\title{
TYPICAL CONVEX SETS OF CONVEX SETS
}

\author{
T. SCHWARZ and T. ZAMFIRESCU
}

(Received 4 March 1986)

Communicated by J. H. Rubinstein

\begin{abstract}
There exists a natural notion of convexity in the space of all compact convex sets in $\mathbb{R}^{d}$. Thus, we may consider the space of all bounded closed convex families of compact convex sets. We present here a strange generic extremal behaviour of the elements of this space.
\end{abstract}

1980 Mathematics subject classification (Amer. Math. Soc.): 52 A 05.

Let $\Re$ denote the space of all compact convex sets of $\mathbb{R}^{d}$, equipped with the Hausdorff distance $\delta$. A set $\mathfrak{H} \subset \mathscr{A}$ is called convex if $A, B \in \mathfrak{A}$ and $\lambda \in[0,1]$ imply $\lambda A+(1-\lambda) B \in \mathscr{A}$ (Minkowski addition). Let $\mathscr{C}$ be the space of all bounded closed convex subsets of $\mathscr{R}$ and let $\Delta$ denote the Hausdorff distance in $\mathscr{C}$. Then $(\mathscr{C}, \Delta)$ is complete ([2], pages 314,315$)$, hence a Baire space too. We always use the words "most" and "typical" in the sense of "all, except those in a set of first Baire category". An element $A \in \mathfrak{A}$ of a set $\mathfrak{A} \in \mathscr{C}$ of convex sets is called extremal if $A$ is not a convex combination of other two elements of $\mathfrak{A}$. As a contribution to the study of the space $\mathscr{C}$, we shall describe in this note the extremal behaviour of typical elements of $\mathscr{C}$.

$V$. Klee (private communication) proved that in an infinite-dimensional compact convex subset $X$ of a normed linear space, most compact convex subsets of $X$ are nowhere dense in $X$. Concerning the extreme points of such sets, Klee [1] showed that in most compact convex subsets of an infinite-dimensional Banach space, the set of extreme points is dense.

The following theorem from [4] is analogous to the first result of Klee mentioned above.

1987 Australian Mathematical Society 0263-6115/87\$A2.00+0.00 
Typical members of $\mathscr{C}$ are nowhere dense in $\Re$.

We establish here the following surprising theorem, similar in spirit to Klee's second result.

\section{THEOREM. In typical members of $\mathscr{C}$ most elements are extremal.}

Proof. We follow the ideas of the proof of Theorem 1 in [3]. Let $B(A, r) \subset \Re$ and $\mathscr{B}(\mathscr{A}, r) \subset \mathscr{C}$ be the balls of radius $r$, and centre $A \in \mathscr{R}$, respectively $\mathfrak{A} \in \mathscr{C}$. Let $S(\mathfrak{A})$ be the set of nonextremal elements of $\mathfrak{A} \in \mathscr{C}$. Then

$$
S(\mathfrak{A})=\bigcup_{n=1}^{\infty} S_{n}(\mathfrak{A})
$$

where $S_{n}(\mathfrak{H})=\left\{\lambda A+(1-\lambda) B: \lambda \in\left[\frac{1}{3}, \frac{2}{3}\right], A, B \in \mathfrak{A}, \delta(A, B) \leqslant 1 / n\right\}$. Let

$$
\begin{aligned}
\mathscr{A} & =\{\mathfrak{U} \in \mathscr{C}: S(\mathfrak{U}) \text { is of second category }\} \\
\mathscr{A}_{n} & =\left\{\mathfrak{A} \in \mathscr{C}: S_{n}(\mathfrak{H}) \text { is not nowhere dense }\right\} \\
\mathscr{A}_{n, m} & =\left\{\mathfrak{A} \in \mathscr{C}: \exists A \in \mathfrak{U} \text { such that } \mathfrak{B}\left(A, m^{-1}\right) \subset \overline{S_{n}(\mathfrak{H})}\right\} .
\end{aligned}
$$

Since

$$
\mathscr{A} \subset \bigcup_{n=1}^{\infty} \mathscr{A}_{n} \subset \bigcup_{n, m=1}^{\infty} \mathscr{A}_{n, m}
$$

it suffices to prove that $\mathscr{A}_{n, m}$ is nowhere dense.

Let $\mathfrak{A} \in \mathscr{C}, \varepsilon>0$ and $\alpha<\varepsilon d^{-1 / 2}$. Consider the lattice $\alpha \mathbb{Z}^{d}$ with fundamental distance $\alpha$ in $\mathbb{R}^{d}$. As every point in $\cup \mathfrak{A}$ has a distance smaller than $\alpha \sqrt{d}$ to some point in $\alpha \mathbb{Z}^{d}$, every element of $\mathfrak{A}$ is at Hausdorff distance smaller than $\alpha \sqrt{d}$ from some polytope with vertices in $\alpha \mathbb{Z}^{d}$. Since $U \mathfrak{A}$ is bounded, all these polytopes are contained in some large ball $K \subset \mathbb{R}^{d}$. The convex hull $\mathfrak{B}$ of the set of all these polytopes (a "polytope of polytopes") is a member of $\mathscr{B}(\mathfrak{A}, \varepsilon)$. We are going to use the fact that the spherical images of the vertices of a polytope in $\mathbb{R}^{d}$, which is a convex combination of two other polytopes, are obtained as intersections of spherical images of vertices of these two polytopes. The set of all spherical images of vertices of polytopes with vertices in $\alpha \mathbb{Z}^{d} \cap K$ is finite and hence so is the set of all spherical images of vertices of elements of $\mathfrak{B}$. Assume now that there is an element $Q_{1} \in \mathfrak{B}$ at distance more than $m^{-1}$ from any extremal element of $\mathfrak{B}$. Consider a polytope $P_{1}$ with $\delta\left(Q_{1}, P_{1}\right)<(2 m)^{-1}$ containing a vertex of spherical image different from and included in one of those mentioned above. Then $P_{1}$ is an extremal element of the convex hull $\mathfrak{B}_{1}$ of $\mathfrak{B} \cup\left\{\boldsymbol{P}_{1}\right\}$ and all extremal elements of $\mathfrak{P}$ are extremal elements of $\mathfrak{B}_{1}$ too. If still there is an element 
$Q_{2} \in \mathfrak{B}_{1}$ at distance more than $m^{-1}$ from any extremal element of $\mathfrak{B}_{1}$, then we insert a new polytope $P_{2}$, and obtain a new set $\mathfrak{B}_{2}$ in the same way. Note that all extremal elements of $\mathfrak{B}_{1}$ are now extremal elements of $\mathfrak{B}_{2}$. We continue this procedure. We claim that it stops after finitely many steps. Suppose, indeed, that we produce infinitely many steps. Then we have a sequence $\left\{P_{n}\right\}_{n=1}^{\infty}$ of constructed polytopes, all in $\mathfrak{B}\left(\cup \mathfrak{B}, m^{-1}\right)$. Then, by Blaschke's theorem, some subsequence converges. But this contradicts the inequality

$$
\delta\left(P_{n}, P_{q}\right) \geqslant \delta\left(P_{q}, Q_{n}\right)-\delta\left(P_{n}, Q_{n}\right)>m^{-1}-(2 m)^{-1}=(2 m)^{-1},
$$

which holds for all $q$ and $n(q<n)$. Hence we can construct a "polytope" $\mathfrak{P}$ ', which is a member of $\mathscr{B}(\mathscr{H}, \varepsilon)-\mathscr{A}_{n, m}$.

Let the polytope $V$ be a fixed extremal element of ' $\mathfrak{B}^{\prime}$. We claim that there exists a ball $\mathcal{O}_{V} \subset \Re$ with centre $V$ such that $\mathcal{O}_{V} \cap S_{n}\left(\mathfrak{P}^{\prime}\right)=\varnothing$. Indeed, suppose we can find a sequence $\left\{A_{i}\right\}_{i=1}^{\infty}$ with $A_{i} \in S_{n}\left(\mathfrak{P}^{\prime}\right)$ converging to $V$. Every $A_{i}$ can be written as $\lambda_{i} P_{i}+\left(1-\lambda_{i}\right) Q_{i}$, with $P_{i}, Q_{i} \in \mathfrak{B}^{\prime}$, and $\frac{1}{3} \leqslant \lambda_{i} \leqslant \frac{2}{3}$. By using Blaschke's theorem and taking an appropriate sequence of indices $\left\{i_{j}\right\}_{j=1}^{\infty}$, we can arrange that $P_{i_{j}} \rightarrow P, Q_{i_{j}} \rightarrow Q$, and $\lambda_{i_{j}} \rightarrow \lambda$, with $P, Q \in \mathfrak{P}^{\prime}$ and $\lambda \in\left[\frac{1}{3}, \frac{2}{3}\right]$. This implies that $V=\lambda P+(1-\lambda) Q$ is not extremal and a contradiction is obtained.

Now we claim that there exists even an open neighbourhood $\mathscr{O}_{V} \subset \mathscr{B}(\mathfrak{A}, \varepsilon)$ of $\mathfrak{B}^{\prime}$ such that $S_{n}(\mathscr{Q}) \cap \mathcal{O}_{V}=\varnothing$ for each $\mathscr{Q} \in \mathcal{O}_{V}$. Indeed, suppose we can find a sequence $\left\{\mathfrak{Q}_{i}\right\}_{i=1}^{\infty}$ converging to $\mathfrak{B}^{\prime}$ such that $S_{n}\left(\mathfrak{Q}_{i}\right) \cap \mathcal{O}_{V} \neq \varnothing$. Choose $B_{i} \in$ $S_{n}\left(\mathfrak{\Omega}_{i}\right) \cap \mathcal{O}_{V}$. We have $B_{i}=\lambda_{i} P_{i}+\left(1-\lambda_{i}\right) Q_{i}$ for suitable $P_{i}, Q_{i} \in \mathfrak{Q}_{i}$ and $\lambda_{i} \in\left[\frac{1}{3}, \frac{2}{3}\right]$. Again we find a sequence of indices $\left\{i_{j}\right\}_{j=1}^{\infty}$ such that $P_{i_{j}} \rightarrow P$, $Q_{i_{j}} \rightarrow Q$, and $\lambda_{i_{j}} \rightarrow \lambda$, with $P, Q \in \mathfrak{B}^{\prime}$ and $\lambda \in\left[\frac{1}{3}, \frac{2}{3}\right]$. Then $B_{i_{j}} \rightarrow B$ for the polytope $B=\lambda P+(1-\lambda) Q$, which belongs to both $S_{n}\left(\mathfrak{P}^{\prime}\right)$ and $\mathcal{O}_{V}$, thus contradicting the choice of $\mathcal{O}_{V}$.

Let $E\left(\mathfrak{P}^{\prime}\right)$ denote the (finite) set of extremal elements of $\mathfrak{P}^{\prime}$ and $\mathcal{O}=$ $\cap_{V \in E\left(\mathfrak{B}^{\prime}\right)} \mathcal{O}_{V}$. We claim the existence of a neighbourhood $\mathscr{N} \subset \mathcal{O}$ of $\mathfrak{B}^{\prime}$ for which the following holds: For every $\mathfrak{Q} \in \mathscr{N}$ and every $B \in \mathfrak{A}$, the ball $\mathfrak{B}\left(B, m^{-1}\right)$ meets the union $\cup_{V \in E\left(\mathfrak{x}^{\prime}\right)}, \mathcal{O}_{V}$. Suppose this is not true. Then there exist a sequence $\left\{\Omega_{i}\right\}_{i=1}^{\infty}$ converging to $\mathfrak{B}^{\prime}$ and a sequence $\left\{B_{i}\right\}_{i=1}^{\infty}$ with $B_{i} \in \Omega_{i}$ such that $\mathfrak{B}\left(B_{i}, m^{-1}\right)$ does not meet $\mathcal{O}_{V}$ for any extremal element $V$ of $\mathfrak{B}^{\prime}$. Again we can select a sequence of indices $\left\{i_{j}\right\}_{j=1}^{\infty}$ such that $\left\{B_{i_{j}}\right\}_{j=1}^{\infty}$ converges. Since $\mathfrak{L}_{i} \rightarrow \mathfrak{B}^{\prime},\left\{B_{i_{j}}\right\}_{j=1}^{\infty}$ converges to an element $P$ of $\mathfrak{B}^{\prime}$. Trivially, $\mathfrak{B}\left(B_{i}, m^{-1}\right) \rightarrow$ $\mathfrak{B}\left(P, m^{-1}\right)$. Hence $\mathfrak{B}\left(P, m^{-1}\right) \cap \mathcal{O}_{V}=\varnothing$ for any $V \in E\left(\mathfrak{B}^{\prime}\right)$, which contradicts the construction of ' $\mathfrak{B}^{\prime}$. This shows that $\mathscr{A}_{n, m}$ is nowhere dense, and the theorem follows. 


\section{Acknowledgement}

During the preparation of this paper both authors enjoyed the hospitality and support of Western Washington University, Bellingham, Washington 98225, U.S.A., which is thankfully acknowledged. They are especially indebted to John Reay for many stimulating discussions.

\section{References}

[1] V. Klee, 'Some new results on smoothness and rotundity in normed linear spaces,' Math. Ann. 139 (1959), 51-63.

[2] C. Kuratowski, Topologie I (Warszawa, 1958).

[3] T. Zamfirescu, 'Many endpoints and few interior points of geodesics', Invent. Math. 69 (1982), 253-257.

[4] T. Zamfirescu, 'Using Baire categories in geometry,' Rend. Sem. Mat. Unil'. Politec. Torino 43 (1985), 67-88.

Department of Mathematics

Ohio State University

Mansfield, Ohio 44906

U.S.A.
Fachbereich Mathematik

Universität Dortmund

46 Dortmund

Federal Republic of Germany 